\title{
Large mucocele with intracranial and intraorbital extension
}

Thanatta Seemai, M.D.

From The Department of Radiology, Faculty of Medicine,

Prince of Songkla University, Songkhla, Thailand

Address correspondence T.S.(e-mail:thanatta.seemai@gmail.com)

\section{Case summary}

A 26-year-old female had had progressive right eye blindness for 13 years. She did not have any other complaints such as headache, seizure, or weakness. At that time she was admitted to a local hospital and Computed tomography (CT) imaging was performed, showing a mass at left maxillary sinus with extracranial extension. The caring physician advised surgery but her family refused due to financial problem. A year later, she developed complete bilateral eye blindness and the subsequent CT revealed larger size of the mass. She was referred to a tertiary hospital for surgery but she lost follow-up. In this visit, she had foul-smelling discharge per left nostril without fever for 2 weeks followed by seizure and headache for 2 days. She had no underlying disease, no history of seizure and head trauma.

Physical examination revealed that her vital signs were normal; body temperature of $36.7^{*} \mathrm{c}$, blood pressure of $100 / 69 \mathrm{mmHg}$, pulse rate of 110 / minute, and respiratory rate of 24/ minute. Both pupils were dilated $(8 \mathrm{~mm} / 8 \mathrm{~mm})$. Light reflexes were diminished in both eyes as well as proptosis with more severity in the left eye. The fundus scope showed bilateral markedly pale discs. There was a mass protruding outside both nostrils. The cranial nerve examination and neurological examinations were normal.

Laboratory investigation for hormonal levels (Free-T4, TSH, HGH cortisol, FSH, LH, prolactin) were all in normal ranges. 


\section{Imaging findings}
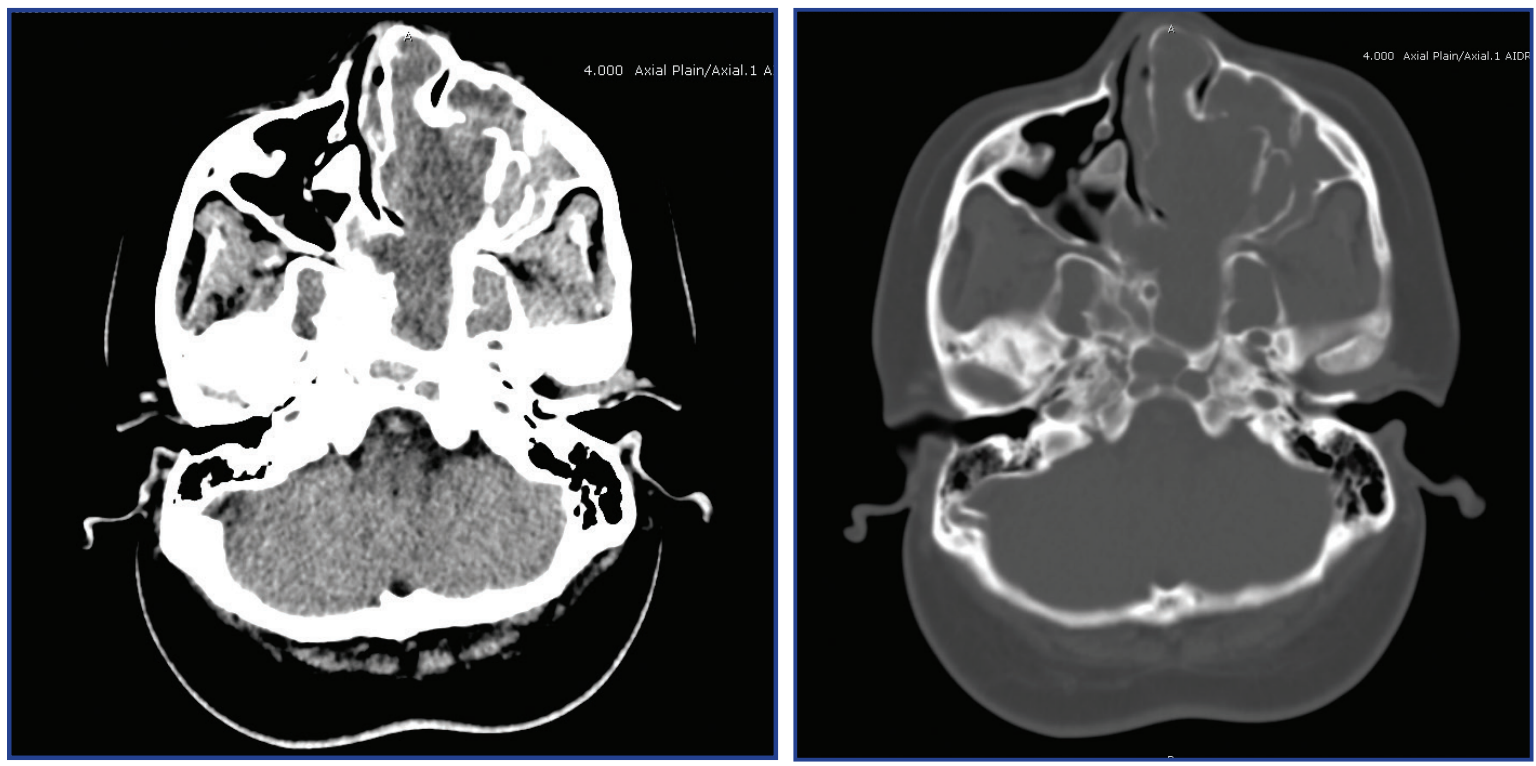

Figure 1. Non-enhanced cranial CT at the level of maxillary sinuses demonstrates large left sinunasal soft tissue with erosion/remodeling and expansion of their bony walls, better demonstrated in bone window (left).
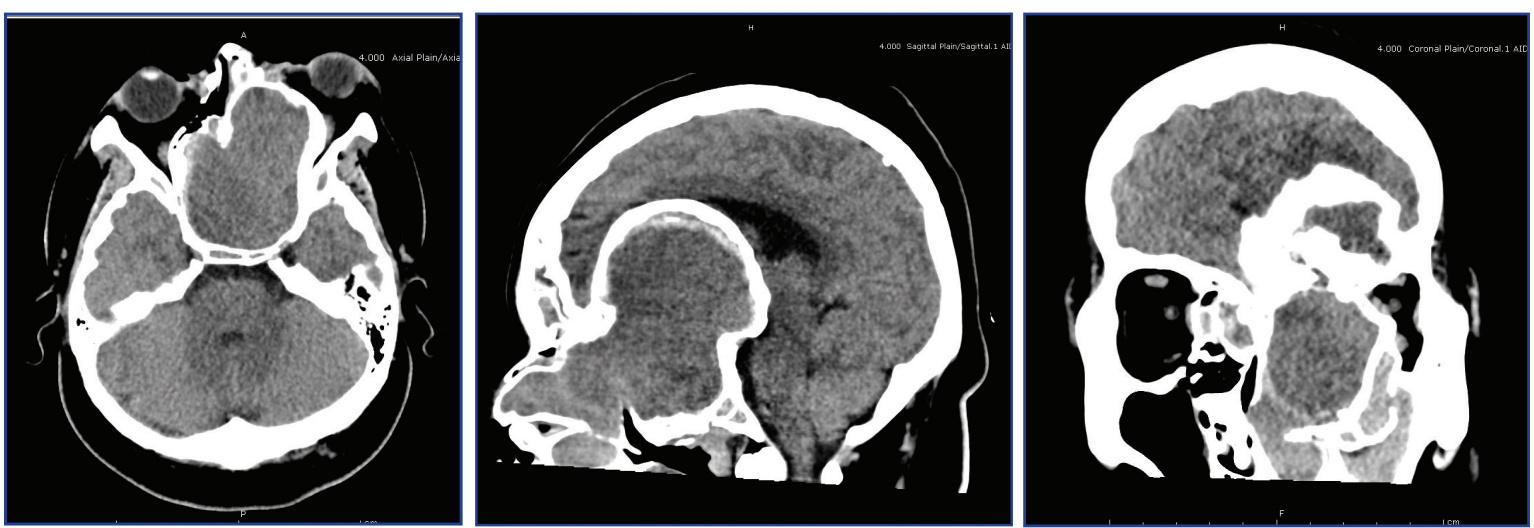

Figure 2. Non-enhanced cranial CT in axial (right), sagittal (middle) and coronal (left) demonstrates a large left sinunasal soft tissue mass with intracranial extension and mass effects causing lateralization of the globe, narrowing retrobulbar space and proptosis. 

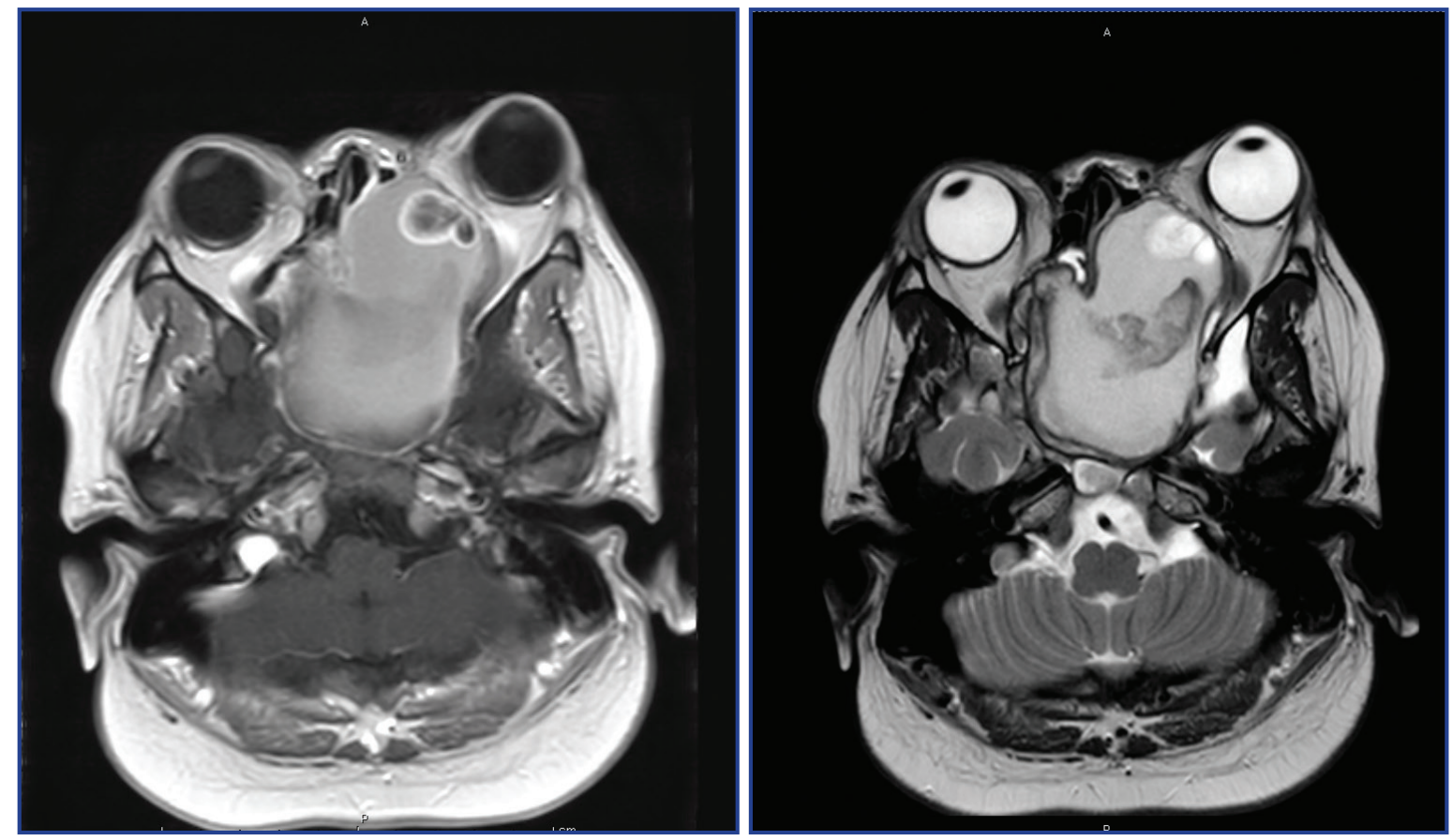

Figure 3. (A) Tl-weighted spin-echo magnetic resonance imaging after intravenous administration of gadolinium demonstrates a non-enhancing mass with hyposignal rim in left sinunasal region with ntracranial extension, displacing adjacent intracranial vessels. (B) The mass appears hyperintense on T2-weighted imaging.

\section{Diagnosis}

A large mucocele with intracranial and intraorbital extension

\section{Treatment}

The patient underwent bilateral tumor resection via an endoscopic sinus surgery (ESS) Intraoperatively, there was an expansile bony tumor with mucoid and soft tissue components occluding bilateral nasal cavities and extending into sphenoethmoidal areas. The mass was removed and pathologically proven to be mucocele.

In spite of complete tumor removal, however, bilateral blindness was still present. The patient was discharged after 11 days of hospitalization. A 6-month follow-up MRI was planned to exclude any recurrence. 


\section{Discussion}

Mucoceles are benign mucus-containing, epithelial lined cystic cavities, developing in paranasal sinuses as a result of chronic obstruction of the outlet ostia that connects the sinus to the nasal cavity.[1,2] They are characterized by a gradual expansion through a continuous mucous-secreting process, resulting in a compression and displacement of the surrounding soft tissues and erosion of the bone walls.[3]

Mucocele could be undiagnosed until symptoms due to compression of surrounding structures arise. The symptoms depend on area of extension: orbital extension (pain, proptosis, loss of vision, ocular motility disturbances, tearing), cranial extension (meningitis, headaches, epidural abscess, subdural empyema, brain abscess, cranial nerve palsies) and nasal expansion (nasal blockage and loss of sense of smell).

Plain skull radiography showing enlarged sinus walls and signs of bone erosion can be suspicious of a mucocele. CT is the most useful investigation in identifying a mucocele, that reveals a non-enhancing airless mass inside the sinus with mucous density.[4] MRI appears iso-hyperintense on T2-weighted imaging. The T1-weighted imaging can vary based on the proportion of mucus, water, and protein contents.[5-7] Showing no contrast enhancement helps differentiate a mucocele from other naso-sinusal tumors. CT scans are much better at delineating the extent of the lesion and its relations to the surrounding structures as compared to an MRI.[8]

Surgery is the required treatment for complete mucocele eradication. This procedure could be achieved with transnasal endoscopic surgery, which has recently become a preferred approach over the more invasive external craniotomy with craniofacial surgery. However, endoscopic transnasal approach is not recommended in cases with intracranial extension due to higher risk of intracavitary residual and post-operative recurrences. $[9,10]$ The prognosis of visual function depends upon the period of visual impairment.

Devars du Mayne et al reported 23.5\% recurrence rate with an average onset of recurrence being at 4 years, more frequently in patients with persistent inflammatory sinus diseases. They advised CT imaging every 2 years for at least 4 to 5 years after surgery. Despite a low recurrence rate, a long follow up is generally recommended.[11,12]

Although most cases in prior reports were largely with/without intracranial extension, few cases were presented with intraorbital extension. One large fronto-orbital mucocele causing proptosis of the right eye and diplopia was reported by Mohan S[13], but the visual acuity was still intact. The other case by Severino $\mathrm{R}$ et al[14], reported a fronto-orbital mucocele with 4-week history of left exophthalmos and sudden proptosis. Both cases had no visual complication after treatment. In this case, we would like to show the rare long-standing large mucocele with serious extension causing poor prognosis in treatment. 


\section{References}

1. Borkar S, Tripathi AK, Satyarthee G, Sharma BS, Mahapatra AK. Frontal mucocele presenting with forehead subcutaneous mass: an unusual presentation. Turk Neurosurg 2008;18:200-3.

2. Lai PC, Liao SL, Jou JR, Hou PK. Transcaruncular approach for the management of frontoethmoid mucoceles. Br J Ophthalmol 2003;87:699-703.

3. Gavioli C, Grasso DL, Carinci F, Amoroso C, Pastore A. Mucoceles of the frontal sinus. Clinical and therapeutical considerations. Minerva Stomatol 2002;51:385-90.

4. Bilaniuk LT, Zimmerman RA. Computer-assisted tomography: sinus lesions with orbital involvement. Head Neck Surg 1980;2:293-301.

5. Kechagias E, Georgakoulias N, Ioakimidou C, Kyriazi S, Kontogeorgos G, Seretis A. Giant intradural mucocele in a patient with adult onset seizures. Case Rep Neurol 2009;1:29-32.

6. Sarsilmaz A, Varer M, Apaydin M, Erdogan N, Uluc E. An unusual presentation of a giant frontal mucocoele manifesting with frontal lobe syndrome. Ann Acad Med Singapore 2009; 38:924-5.

7. Capra GG, Carbone PN, Mullin DP. Paranasal sinus mucocele. Head Neck Pathol 2012; 6:369-72.

8. Bilaniuk LT, Zimmerman RA. Computer-assisted tomography: sinus lesions with orbital involvement. Head Neck Surg 1980;2:293-301.

9. Pia F, Aluffi P, Borello G. [Frontal mucocele: open surgery]. Acta Otorhinolaryngol Ital 1999;19:155-9. Italian.

10. Wang KW, Hsu HC, Lu K, Chen HJ, Liang CL. Outer table craniotomy for frontal sinus mucocele. J Craniofac Surg 2004;15:869-73; discussion 873-4.

11. Constantinidis J, Steinhart H, Schwerdtfeger K, Zenk J, Iro H. Therapy of invasive mucoceles of the frontal sinus. Rhinology 2001;39:33-8.

12. Weitzel EK, Hollier LH, Calzada G, Manolidis S. Single stage management of complex fronto-orbital mucoceles. J Craniofac Surg 2002;13:739-45.

13. Mohan S. Frontal sinus mucocele with intracranial and intraorbital extension: a case report. J Maxillofac Oral Surg 2012;11:337-9.

14. Severino R, Severino P. Fronto-orbital mucocele with intracranial extension: a case report. J Surg Case Rep 2017;2017:rjx107. doi:10.1093/jscr/rjx107. 